\title{
Seeing the full picture of metabolism
}

Cells, tissues and organisms contain complex mixtures of chemicals, the products and by-products of metabolism. Although mass spectrometry was routinely used to identify specific classes of metabolites starting in the mid-1950s (Milestone 7), it would take another decade before researchers began using the technique to examine complex metabolite mixtures.

In one early example, E.C. Horning and colleagues separated and characterized organic compounds from urine, mainly acids and steroids, using gas-liquid chromatography and confirming chemical identity by mass spectrometry. In 1971, they extended their analysis to include drugs and drug metabolites, initiating the use of the method as a means of establishing a 'metabolic profile' of an individual.

Despite these early instances of complex metabolite identification, the field lay relatively dormant for more than 20 years, until Richard Gross and colleagues reported the identification of more than 50 phospholipids from erythrocyte membranes in 1994. Several other groups later reported the identification of complex lipid mixtures or the quantification of a few select species, and the identification and quantification of phospholipids from total cellular lipid extracts in 1997 by F.T. Wieland, W.D. Lehmann and colleagues heralded the birth of lipidomics. By using electrospray ionization mass spectrometry (ESIMS) (Milestone 15), optimizing fragmentation energies for each phospholipid headgroup class and including internal standards with non-natural fatty acids, they were able to assess both the quality and quantity of a lipid extract.

Metabolic profiling by mass spectrometry became part of the functional genomics toolkit in 2000 when Oliver Fiehn and colleagues compared extracts from two Arabidopsis thaliana ecotypes, finding that they expressed distinct metabolic profiles. It was not long before this approach was applied to yeast, and later adapted to a high-throughput format by analyzing spent culture medium rather than cell lysate, a method Douglas Kell and colleagues referred to as 'metabolic footprinting'. In 2002, Ralf Takors and colleagues described a process to monitor the flow of metabolites in Escherichia coli in response to changes in nutrient status, which they termed 'metabolomics'. Whereas mass spectrometry had previously been used to present static snapshots of metabolic state, now dynamic changes could be monitored. The following year, Eliane Fischer and Uwe Sauer's investigation of the metabolic flux of carbon through a panel of $E$. coli mutants provided insight into central carbon metabolism in this organism. More recent metabolomics studies yield increasingly complicated datasets, whose interpretation has been aided by improvements in mass spectrometry techniques and by software tools such as XCMS designed to enable facile detection of unknown spectrum peaks that differ substantially in concentration across samples.

Following on from its early use in profiling complex metabolite mixtures in biological fluids,

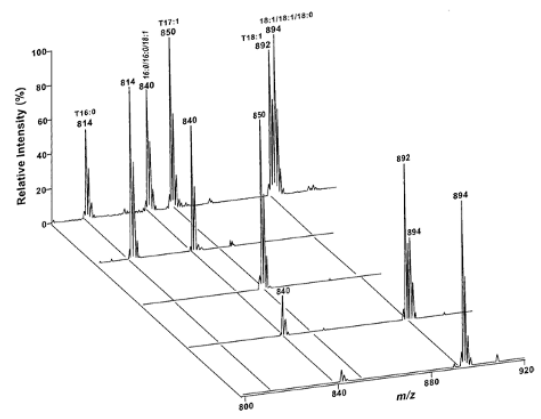

Identification of triglycerides in a mixture by ESI-MS and tandem mass spectrometry. Image reproduced from Han, X. \& Gross, R.W., Anal. Biochem. 295, 88-100 (2001), with permission from Elsevier. metabolomics has recently found an expanding number of uses in basic research and medicine, from gaining insight into how metabolic pathways interact and influence one another to understanding the effect of drugs and diet on our bodies. Applying metabolomics to direct synthetic biology approaches and adapting it to advance personalized medicine are two of the current challenges and opportunities facing metabolomics researchers.

Kyle Legate, Associate Editor, Nature Communications

ORIGINAL RESEARCH PAPERS Dalgliesh, C.E., Horning, E.C Horning, M.G., Knox, K.L. \& Yarger, K. Gas-liquidchromatographic procedure for separating a wide range of metabolites occurring in urine or tissue extracts. Biochem. $\mathrm{J}$ 101, 792-810 (1966) | Horning, E.C. \& Horning, M.G. Metabolic profiles: gas-phase methods for analysis of metabolites. Clin. Chem. 17, 802-809 (1971) | Han, X. \& Gross, R.W. Electrospray ionization mass spectroscopic analysis of human erythrocyte plasma membrane phospholipids. Proc. Natl. Acad. Sci. USA 91, 10635-10639 (1994) | Brügger, B., Erben, G., Sandhoff, R., Wieland, F.T. \& Lehmann, W.D. Quantitative analysis of biological membrane lipids at the low picomole level by nano-electrospray ionization tandem mass spectrometry. Proc. Natl. Acad. Sci. USA 94, 2339-2344 (1997) | Fiehn, O. et al. Metabolite profiling for plant functional genomics. Nat. Biotechnol. 18, 1157-1161 (2000) | Buchholz, A. Hurlebaus, J., Wandrey, C. \& Takors, R. Metabolomics: quantification of intracellular metabolite dynamics. Biomol. Eng. 19, 5-15 (2002)

FURTHER READING McFadden, W.H., Teranishi, R., Corse, J. Black, D.R. \& Mon, T.R. Volatiles from strawberries. II. Combined mass spectrometry and gas chromatography on complex mixtures. J. Chromatogr. 18, 10-19 (1965) | Allen, J. et al. High-throughput classification of yeast mutants for functional genomics using metabolic footprinting. Nat Biotechnol. 21, 692-696 (2003) | Fischer, E. \& Sauer, U. Metabolic flux profiling of Escherichia coli mutants in central carbon metabolism using GC-MS. Eur. J. Biochem. 270, 880891 (2003) | Smith, C.A., Want, E.J., O'Maille, G., Abagyan, R. \& Siuzdak, G. XCMS: processing mass spectrometry data for metabolite profiling using nonlinear peak alignment, matching, and identification. Anal. Chem. 78, 779-787 (2006) 